\title{
Prevalence of Echinococcus Species in Wild Foxes and Stray Dogs in Qinghai Province, China
}

\author{
Huixia Cai, ${ }^{1} \dagger$ Jing Zhang, ${ }^{2} \dagger$ Xuefei Zhang, ${ }^{1}$ Yayi Guan, ${ }^{2}$ Xiao Ma, ${ }^{1 \star}$ Jianping Cao, ${ }^{2}$ Junying Ma, ${ }^{1}$ Na Liu, ${ }^{1} \mathrm{Hao}$ Wu, ${ }^{3}$ Yufang Liu, ${ }^{1}$ \\ Jia Liu, ${ }^{1}$ Wei Wang, ${ }^{1}$ Wen Lei, ${ }^{1}$ Kemei Shi, ${ }^{1}$ Qing Zhang, ${ }^{1}$ Xiongying Zhang, ${ }^{1}$ Peizhen Zhan, ${ }^{1}$ and Yujuan Shen ${ }^{2 *}$ \\ ${ }^{1}$ Department of Parasite Control, Qinghai Province Institute for Endemic Diseases Prevention and Control, Xining, China; ${ }^{2}$ National Institute of \\ Parasitic Diseases, Chinese Center for Disease Control and Prevention (Chinese Center for Tropical Diseases Research), NHC Key Laboratory of \\ Parasite and Vector Biology, WHO Collaborating Center for Tropical Diseases, National Center for International Research on Tropical Diseases, \\ Shanghai, China; ${ }^{3}$ Department of Medical Record Information, Qinghai Provincial Traffic Hospital, Xining, China
}

\begin{abstract}
Echinococcosis is a zoonotic parasitic disease that is highly endemic to the Qinghai province of China. Limited data are available on the prevalence of the causal pathogen, Echinococcus spp., in definitive hosts in this region. Thus, the aim of this study was to evaluate the prevalence of Echinococcus spp. in wild foxes and stray dogs in Qinghai province. Five hundred and twenty-eight feces from wild foxes and 277 from stray dogs were collected from 11 counties in the Golog, Yushu, and Haixi prefectures and screened for Echinococcus spp. using copro-DNA polymerase chain reaction (PCR). In total, $5.5 \%$ of wild foxes and $15.2 \%$ of stray dogs tested positive for Echinococcus spp. The prevalence rates of Echinococcus spp. in wild foxes in Golog, Yushu, and Haixi were 7.3\%, 5.2\%, and 1.9\%, respectively. In stray dogs, these rates were $13.3 \%, 17.3 \%$, and $0 \%$, respectively. Sequencing analysis determined that Echinococcus multilocularis was the most prevalent species, occurring in $4.0 \%$ and $12.6 \%$ of wild foxes and stray dogs, respectively. Echinococcus shiquicus was observed in $1.5 \%$ of wild foxes and $0.7 \%$ of stray dogs. Echinococcus granulosus was observed only in wild dogs, with a prevalence rate of $1.8 \%$. To our knowledge, this is the first report on the prevalence of E. shiquicus in dogs in Qinghai province. The current results improve our understanding of the transmission and dissemination of human echinococcosis and suggest that exposure to the eggs of $E$. multilocularis harbored by wild foxes and stray dogs may pose a great risk of alveolar echinococcosis to humans in Qinghai province.
\end{abstract}

\section{INTRODUCTION}

Echinococcosis, caused by metacestodes of the genus Echinococcus, is a chronic zoonotic disease endemic around world. ${ }^{1}$ In the most recent taxonomic revision, nine species were recognized in the genus Echinococcus, including Echinococcus granulosus sensu stricto (Eg; genotypes G1-G3), E. multilocularis (Em), E. oligarthra, E. vogeli, E. shiquicus (Es), E. equinus (G4), E. ortleppi (G5), E. canadensis (G6-G10) (Ec), and E. felidis. ${ }^{2} \mathrm{Eg}$, Em, Ec, and Es have been isolated from animal hosts in China, whereas $\mathrm{Eg}, \mathrm{Em}$, and $\mathrm{Ec}$ have been detected in humans. ${ }^{3,4} \mathrm{Eg}$ and $\mathrm{Em}$ cause cystic echinococcosis (CE) and alveolar echinococcosis (AE), respectively, in humans. Cystic echinococcosis and $\mathrm{AE}$ are significant threats to public health in the pastoral areas of western and northwestern China. ${ }^{5}$

Qinghai Province is one of the areas within China where echinococcosis (CE and AE) is highly endemic. ${ }^{6,7}$ The Golog Tibetan Autonomous Prefecture (Golog) and Yushu Tibetan Autonomous Prefecture (Yushu) of the southern plateau region in Qinghai are the most severely affected, with echinococcosis prevalence ranging from $0.2 \%$ to $12.38 \%$ for 12 Tibetan counties across these prefecture in $2012 .{ }^{8}$ Further, the Golog had the highest average prevalence of human echinococcosis $(5.2 \%),{ }^{9}$ whereas in Yushu it was $4.54 \% .^{10}$ The prevalence ranges for counties within these two Tibetan

*Address correspondence to Xiao Ma, Department of Parasite Control, Qinghai Province Institute for Endemic Diseases Prevention and Control, Xining 811602, China, E-mail: maxiao0971@163.com or Yujuan Shen, National Institute of Parasitic Diseases, Chinese Center for Disease Control and Prevention (Chinese Center for Tropical Diseases Research), NHC Key Laboratory of Parasite and Vector Biology, Shanghai 200025, China, E-mail: shenyj@nipd.chinacdc.cn.

†These authors contributed equally to this work. autonomous prefectures were $0.2-8.2 \% \quad(A E)$ and 2.62-6.11\%(CE), respectively..$^{11-16}$ However, the Haixi Menggu and Tibetan Autonomous Prefecture (Haixi), neighbors of Yushu and Golog, exhibited relatively low echinococcosis prevalence, and only CE has been reported there. ${ }^{17}$

Echinococcosis is transmitted via carnivore definitive and herbivore/omnivore intermediate hosts through predator-prey interactions, which results in different life cycles types, such as domestic, wildlife, or hybrid. Eg is predominately transmitted among dogs and livestock, whereas the transmission cycles of Em and Es involve dogs and wild foxes as the primary definitive hosts, and a variety of small mammals as intermediate hosts. Humans are typically infected with Echinococcus spp. through direct contact with feces from infected animals, or via the consumption of contaminated food and water. In Tibetan areas, domestic and stray dogs are the biggest contributors to CE and AE prevalence, whereas wild foxes are the primary source of $A E .{ }^{18,19}$ In recent years, there have been more investigations on domestic dogs, ${ }^{9,10,14,20}$ and limited research on nondomesticated carnivores. Studying the latter group is critical for understanding the risk posed to humans.

In the present study, we investigated the prevalence of Echinococcus spp. in wild foxes and stray dogs in Golog, Yushu, and Haixi prefectures of Qinghai province using copro-DNA polymerase chain reaction (PCR) analysis. The current findings will contribute to the development of prevention and control strategies for echinococcosis in these areas.

\section{MATERIALS AND METHODS}

Study regions. Study regions were selected based on well-documented high prevalence and geography. The regions evaluated in this study are shown in Figure 1. 


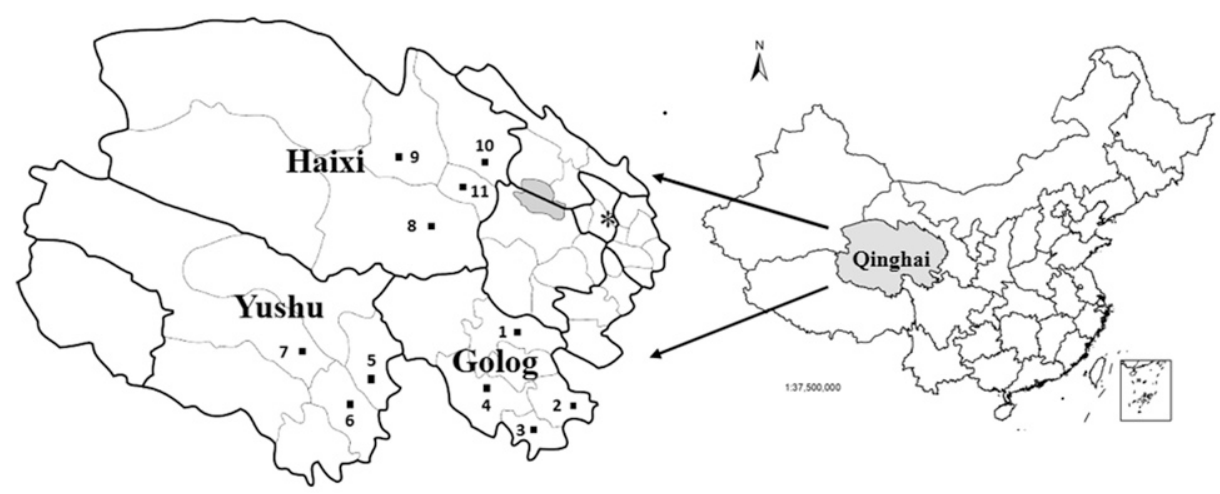

FIGURE 1. Study areas in Qinghai province, China. *Xining, provincial capital; sites 1-11, samples collected from 11 counties across three prefectures.

Golog (longitude: $97^{\circ} 54^{\prime}-120^{\circ} 50^{\prime}$; latitude: $32^{\circ} 31^{\prime}-35^{\circ} 40^{\prime}$ ) is a pastureland area in the Qinghai-Tibet Plateau, with a mean altitude of $4,200 \mathrm{~m}$ above sea level, and an annual mean temperature of $1.1^{\circ} \mathrm{C}$. There are a large number of canids, including domestic and stray dogs, foxes, and wolves, as well as $>250,000$ livestock. Fecal samples were collected from four counties (Maqin, Dari, Jiuzhi, and Banma) in 2015.

Yushu (longitude: $89^{\circ} 27^{\prime}-97^{\circ} 39^{\prime}$; latitude: $31^{\circ} 45^{\prime}-36^{\circ} 10^{\prime}$ ) is a pastureland area located in the Qinghai-Tibet Plateau, adjacent to Haixi and Golog. Similar to Golog, Yushu has a mean altitude of $4,200 \mathrm{~m}$ above sea level, with an annual mean temperature of $3.3^{\circ} \mathrm{C}$. Large numbers of livestock and wild canids inhabit this area. Fecal samples were collected from three counties (Yushu, Chenduo, and Zhiduo) in 2014.

Haixi is located in the west of Qinghai province and north of the Qinghai-Tibet Plateau (longitude: $90^{\circ} 06^{\prime}-99^{\circ} 42^{\prime}$; latitude: $\left.35^{\circ} 01^{\prime}-39^{\circ} 20^{\prime}\right)$, at the southern border of Golog and Yushu. It comprises part of the Qaidam Basin, most of the Gobi Desert, marshes, and saltwater lakes, with a terrain typical of the highest plateau inland basins. The mean altitude of the basins is $3,000 \mathrm{~m}$ above sea level, and the annual mean temperature is $5.2^{\circ} \mathrm{C}$, with a continental arid climate. The economy of Haixi drives by industries, with a minor contribution from agriculture and animal husbandry. Fecal samples were collected from four counties (Delinha, Wulan, Dulan, and Tianjun) in 2015.

Specimen collection. In the 11 investigated counties, we determined a collection area at every $2 \mathrm{~km}$ or more along the road in each county, depending on the surrounding environment, the distribution of rat holes, the haunt of the foxes and stray dogs, and so on. At least a dozen such collection areas were designated in each county. Wild fox feces were identified by shape, color, moisture, and smell, and were collected from rodent holes/mounds, soddy walls, and ditches. Stray dog feces were collected from inhabited areas of the Golog and Yushu prefectures, but not from Haixi, where stray dogs are extremely rare. To minimize the repeated sampling of feces from the same animal, each samples was collected at a minimal distance of 200-300 m from the previous. Each sample was individually collected in a $50-\mathrm{mL}$ centrifuge tube and stored at $-80^{\circ} \mathrm{C}$ for at least 1 week to inactivate the eggs of Echinococcus spp. ${ }^{21}$

DNA extraction and host species identification. Total genomic DNA was extracted from fecal samples (200-300 mg) using the QIAamp DNA Stool Mini Kit (Qiagen, Hilden, Germany) according to the manufacturer's instructions. The inhibitEX tablets and Buffer ASL from the Kit efficiently adsorbed substances in the fecal samples that may degrade DNA from the fecal samples. The extracted genomic DNA samples were stored at $-20^{\circ} \mathrm{C}$. To identify the origin species in the fecal samples, the primer pair H15149L/L14724 was used to amplify cytochrome b (729 bp) mitochondrial DNA of the family Canidae via copro-DNA PCR, as previously described. ${ }^{22}$ All DNA samples were amplified at least three times. All PCR products were sequenced by Shanghai Biotechnology Co. (Shanghai, China) and compared against sequences in the NCBI database. Only DNA samples extracted from foxes and dogs were used for Echinococcus spp. identification.

Parasite identification. Em and Es were identified using nested-PCR for the mitochondrial cytochrome c oxidase subunit I (coxl) gene, ${ }^{23}$ and Eg was identified using speciesspecific primers for the mitochondrial NADH dehydrogenase subunit I (nadl) by PCR, as previously described. ${ }^{24-27}$ Polymerase chain reaction protocols are described in Table 1. For all PCRs, distilled water was used as a negative control, and DNA from the adult worms of Em, Es, and $\mathrm{Eg}$ was used as positive controls. Each DNA sample was amplified at least three times. PCR products were visualized using 1.5\% agarose gel electrophoresis with ethidium bromide staining. Positive PCR products were sequenced by Shanghai Biotechnology Co. Each sequence was compared against sequences in the NCBI database to determine the species of Echinococcus spp.

Statistical analysis. Data were analyzed using SPSS software (SPSS Inc., Chicago, IL) and mapped using ArcGIS 10.1 (ESRI, RedLands). Differences in the presence of Echinococcus spp. were evaluated with the $\chi^{2}$ test. $P<0.05$ was considered to indicate statistically significance. The Wilson score method was used to calculate the $95 \% \mathrm{Cl}$ for each group of proportions.

\section{RESULTS}

Prevalence of Echinococcus spp. in wild foxes and stray dogs. Of the 805 collected fecal samples, 528 were from wild foxes, and 277 were from stray dogs. Twenty-nine samples from wild foxes were positive for Echinococcus spp. (5.5\%, 95\% Cl: 3.9-7.8). Among these, 21 contained 
TABLE 1

Summary of PCR protocols for species identification

\begin{tabular}{|c|c|c|c|c|c|}
\hline Target & Gene & Step & Primers & Product (bp) & Method \\
\hline Taeniidae $^{25}$ & Coxl (external) & First & $\begin{array}{l}\text { FP:5'TTGAATTTGCCACGTTTGAATGC-3' } \\
\text { RP:5'GAACCTAACGACATAACATAATGA-3' }\end{array}$ & 874 & Nested-PCR \\
\hline $\begin{array}{l}\text { Echinococcus } \\
\text { multilocularis }^{26}\end{array}$ & Coxl (internal) & Second & $\begin{array}{l}\text { FP:5'GTCATATTTGTTTAAGTATAAGTGG-3' } \\
\text { RP:5'CACTCTTATTTACACTAGAATTAAG-3' }\end{array}$ & 243 & \\
\hline $\begin{array}{l}\text { Echinococcus } \\
\text { shiquicus }^{27}\end{array}$ & Coxl (internal) & Second & $\begin{array}{l}\text { FP:5'GTTGGTTACGTTACCGGTT-3' } \\
\text { RP:5'-TCTTATTAACATTTGAATTCAAC-3' }\end{array}$ & 420 & \\
\hline $\begin{array}{l}\text { Echinococcus } \\
\text { granulosus }^{24}\end{array}$ & $\mathrm{Nadl}$ & First & $\begin{array}{l}\text { FP:5'GGTTTTATCGGTATGTTGGTGTTAGTG-3' } \\
\text { RP:5'CATTTCTTGAAGTTAACAGCATCACG-3' }\end{array}$ & 219 & Ordinary PCR \\
\hline
\end{tabular}

Em (4.0\%, 95\% Cl: 2.6-6.0), and eight contained Es (1.5\%, 95\% Cl: 0.8-3.0). Forty-two stray dogs were positive for Echinococcus spp., with a prevalence of $15.2 \%(95 \% \mathrm{Cl}$ : 11.4-19.9, 42/277). Of these, 35 contained Em (12.6\%, 95\% Cl: 9.2-17.1), two contained Es (0.7\%, 95\% Cl: 0.2-2.6), and five contained $\mathrm{Eg}(1.8 \%, 95 \% \mathrm{Cl}$ : 0.8-4.1). All Eg-positive samples were identified as of the G1 genotype, based on sequencing of the nadl gene. The general prevalence rates of Echinococcus spp. $\left(\chi^{2}=21.13, P<0.001\right)$ and the prevalence of specific Echinococcus species $\left(\chi^{2}=31.89\right.$, $P<0.001)$ differed significantly between the two hosts (Figure 2). No animals were infected by more than one Echinococcus species.

Prevalence of Echinococcus spp. by geographic region. In Golog, 246 wild fox fecal samples and 150 stray dog fecal samples were collected. In Yushu, 174 wild fox fecal samples and 127 stray dog fecal samples were collected. About 108 wild fox fecal samples were collected from Haixi. The prevalence rate of Echinococcus spp. in wild foxes was 7.3\% in Golog, 5.2\% in Yushu, and 1.9\% in Haixi, differing significantly among the three prefectures $\left(\chi^{2}=\right.$ 11.49, $P<0.05)$. The prevalence rates of Echinococcus spp. among stray dogs were $13.3 \%$ and $17.3 \%$ in Golog and Yushu, respectively, with no significant differences between the prefectures $\left(\chi^{2}=5.43, P>0.05\right)$. Significant differences in the prevalence of Echinococcus spp. were observed between wild foxes and stray dogs in Yushu $\left(\chi^{2}=20.91\right.$, $P<0.001)$ and in Golog $\left(\chi^{2}=7.81, P<0.05\right)$.

In Golog, 16 (6.5\%) wild fox fecal samples were positive for Em and two (0.8\%) for Es. Of the nine fecal samples from wild foxes in Yushu, which were positive for Echinococcus spp., five $(2.9 \%)$ contained Em, and four (2.3\%) contained

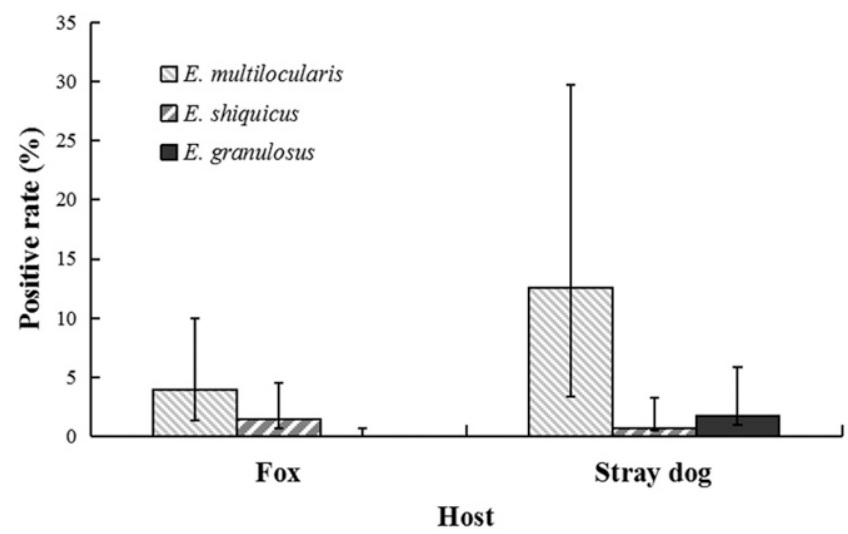

FIGURE 2. Prevalence of Echinococcus species in fecal samples from wild foxes and stray dogs.
Es. In Haixi, two Echinococcus spp.-positive wild fox fecal samples (1.9\%) contained Es, while Em was not detected. None of the fecal samples from wild foxes collected across the three prefectures tested positive for Eg. The prevalence rate of Es among wild fox fecal samples was not significantly different across the three prefectures $\left(\chi^{2}=1.61, P>0.05\right)$. Em occurrence rates among wild fox fecal samples were significantly different between Golog and Haixi $\left(\chi^{2}=7.36\right.$, $P<0.01)$. In addition, Em was identified in 9.3\% (14/150) and $16.5 \%(21 / 127)$ of fecal samples from stray dogs in Golog and Yushu, respectively, and these rates were not significantly different $\left(\chi^{2}=3.23, P>0.05\right)$. Furthermore, four stray dog fecal samples from Golog (4/150, 2.7\%) and one from Yushu (1/127, 0.8\%) were positive for Eg (not significantly different: $\left.\chi^{2}=1.37, P>0.05\right)$. Es was also detected in stray dog fecal samples from Golog (2/150, 1.3\%) (Table 2).

\section{DISCUSSION}

The prevention and control of echinococcosis in Qinghai province is a long-term and arduous task. Echinococcosis was prevalent in 39 out of 43 counties in Qinghai Province, covering a total area of $650,000 \mathrm{~km}^{2}$ with $A E$ and CE coexisting in 14 counties. The most severely affected area is the southern part of Qinghai province, where the population density is 1.8 person $/ \mathrm{km}^{2}$. These area located in highaltitude pastures, where local residents engage in seminomadic livestock production activities, thus being more closer to domestic and wild canids and therefore are at a greater risk of contracting echinococcosis.

Dogs were identified as a major source of human echinococcosis infection in a previous study. ${ }^{28}$ Twenty years ago, the prevalence rate of Echinococcus spp. among domestic dogs in Qinghai province was $40 \%$, with rates up to $70 \%$ in Golog and Yushu. ${ }^{29}$ In 2006-2015, the National Echinococcosis Control Project adopted a program for deworming domestic dogs (using praziquantel) as a substantial control measure, that was incrementally carry out from three to 39 counties in Qinghai province. In 2012, the prevalence of Echinococcus infection among domestic dogs was $13.02 \%$. $^{8}$ Thus, the prevalence decreased significantly from $40 \%$ to $13.02 \%$, owing to the above-described deworming strategy. In 2010, the implementation of the National Echinococcosis Control Project has gradually expanded from a single health department to 14 departments including agriculture, forestry and grassland, water resources and public security, and so on. ${ }^{30}$ Since then, endemic counties across Qinghai have started to implement measures such as domestic dog 
TABLE 2

Prevalence of Echinococcus species in wild foxes and stray dogs in Qinghai province

\begin{tabular}{|c|c|c|c|c|c|c|}
\hline \multirow[b]{2}{*}{ Host } & \multirow[b]{2}{*}{ Location } & \multirow[b]{2}{*}{ Number of samples } & \multicolumn{3}{|c|}{ Number of positive samples (\%/95\% Cl) } & \multirow{2}{*}{$\begin{array}{l}\text { Total number of positive samples } \\
\qquad(\% / 95 \% \mathrm{Cl})\end{array}$} \\
\hline & & & Echinococcus multilocularis & Echinococcus shiquicus & Echinococcus granulosus & \\
\hline \multirow[t]{3}{*}{ Fox } & Golog & 246 & $16(6.5 / 4.0-10.3)$ & $2(0.8 / 0.2-2.9)$ & $0(0 / 0.0-1.5)$ & $18(7.3 / 4.7-11.3)$ \\
\hline & Haixi & 108 & $0(0 / 0.0-3.4)$ & $2(1.9 / 0.5-6.5)$ & $0(0 / 0.0-3.4)$ & $2(1.9 / 0.5-6.5)$ \\
\hline & Yushu & 174 & $5(2.9 / 1.2-6.6)$ & $4(2.3 / 0.9-5.8)$ & $0(0 / 0.0-2.16)$ & $9(5.2 / 2.7-9.5)$ \\
\hline \multirow[t]{2}{*}{ Stray dog } & Golog & 150 & $14(9.3 / 5.6-15.1)$ & $2(1.3 / 0.4-4.7)$ & $4(2.7 / 1.0-6.7)$ & 20 (13.3/8.8-19.7) \\
\hline & Yushu & 127 & $21(16.5 / 11.1-24.0)$ & $0(0 / 0.0-2.9)$ & $1(0.8 / 0.1-4.3)$ & $22(17.3 / 11.7-24.8)$ \\
\hline
\end{tabular}

registration and management, partial-stray dog aperiodic reception or disposal, livestock immunization and slaughter management, grassland management, rodent control, and so on. In fact, 10 years ago, stray dogs and unleashed domestic dogs were ubiquitous in all counties of Qinghai Province. Since 2010, the number of stray dogs has been significantly reduced. This was also reflected by our study, where the number of fecal samples collected from stray dogs was less than that from the foxes. It is worth noting that the wild foxes and stray dogs could still be observed in and around human settlements during our survey in 2014-2015, although wild foxes is common far from villages and the some stray dog were also adopted or disposed irregularly. Their feces, which contain eggs, can therefore contaminate surroundings, posing a significant risk of infection to local residents, herders, or travelers.

Control programs have certainly had an impact on the prevalence of Echinococcus in stray dogs and wild foxes. Compared with data of stray dogs in the Qinghai southern plateau from studies conducted during the 1995-2010 period, ${ }^{31}$ the prevalence rates of $\mathrm{Em}$ and $\mathrm{Eg}$ among stray dogs in Golog and Yushu determined in the current study were lower. Furthermore, the prevalence rates of Em in wild foxes were lower compared with data of wild foxes in Qinghai southern plateau. There may be various reasons for this difference, including deworming measure, the prohibition of unleashed domestic dogs, as well as the implementation of grassland rodent and partial-stray dog aperiodic reception or disposal measures. Our study found that the prevalence of Echinococcus in stray dogs was higher than wild foxes, which was in agreement with a report from $2018 .^{32}$ Further, the prevalence of Echinococcus in stray dogs was similar to that in domestic dogs in Qinghai Province (13.02\%). These data indicate that stray dogs are the major source of infection in the wildlife transmission cycle, followed by wild foxes. The level of Echinococcus infection in stray dogs is similar to that in domestic dogs, suggesting that both represent important targets for echinococcosis control in the future. In the current study, the prevalence of Eg in stray dogs was significantly lower than $\mathrm{Em}$. The $\mathrm{Eg}$ infection prevalence among stray dogs in Golog and Yushu was lower than that of previously reports for the Qinghai southern plateau, possibly because of the recent enforcement of livestock slaughter regulations. This further suggests that stray dogs might feed

TABLE 3

Worldwide prevalence of Echinococcus spp. in foxes and dogs over the last 3 years

\begin{tabular}{|c|c|c|c|c|}
\hline Country & Location & Host & Positive no./sample no. (\%) & $\begin{array}{l}\text { Echinococcus species } \\
\text { (no. positive samples) }\end{array}$ \\
\hline \multirow[t]{9}{*}{ China } & Ningxia $^{36}$ & Domestic dog & 250/750 (33.3) & $\begin{array}{l}\text { Em (106); Eg (124); } \\
\quad \text { mixed Em and Eg (20) }\end{array}$ \\
\hline & \multirow[t]{2}{*}{ Qinghai* $^{*}$} & Wild fox & 29/528 (5.5) & Em (21); Es (8) \\
\hline & & Stray dog & $42 / 277$ (15.2) & Em (35); Es (2); Eg (5) \\
\hline & Qinghai $^{20}$ & Domestic dog & $36 / 144(25.0)$ & $\begin{array}{l}\text { Eg (35); mixed Em and } \\
\text { Eg (1) }\end{array}$ \\
\hline & \multirow[t]{2}{*}{ Qinghai $^{32}$} & Wild fox & $6 / 161(3.7)$ & - \\
\hline & & Stray dog & 8/61 (13.1) & - \\
\hline & Sichuan $^{37}$ & Domestic dog & $11 / 120(9.2)$ & Em \\
\hline & Tibet $^{38}$ & Domestic dog & $552 / 7,564(7.3)$ & - \\
\hline & Xinjiang 39 & Domestic dog & $74 / 2,219$ (3.3) & - \\
\hline Bhutan & Tsirang/Gelephu ${ }^{40}$ & Stray dog & $10 / 138(7.3)$ & $\mathrm{Eg}$ \\
\hline Canada & Ontario 41 & Wild fox & $9 / 44(20.5)$ & $\mathrm{Em}$ \\
\hline France & Corsica $^{42}$ & Domestic dog & $3 / 259(1.2)$ & Ec \\
\hline India & Maharashtra $^{43}$ & Stray/domestic dog & $19 / 289(6.6)$ & - \\
\hline Iran & Kerman ${ }^{44}$ & Stray dog & $34 / 307$ (11.1) & Eg (21); others (13) \\
\hline Kenya & $\begin{array}{l}\text { Turkana/lsiolo/Meru/ } \\
\text { Narok }^{45}\end{array}$ & Domestic dog & $71 / 1,621(4.4)$ & $\begin{array}{l}\text { Eg (43); Ec (15); Eo (4); } \\
\text { mixed Eg and Ec (7); } \\
\text { mixed Eg and Eo (1); } \\
\text { mixed Eg, Ec and } \\
\text { Eo (1) }\end{array}$ \\
\hline \multirow[t]{2}{*}{ Poland } & \multirow[t]{2}{*}{ Podkarpackie 46} & Domestic dog & $4 / 268(1.5)$ & $\mathrm{Em}$ \\
\hline & & Wild fox & $53 / 110$ (48.2) & $\mathrm{Em}$ \\
\hline Serbia & Vojvodina $^{47}$ & Wild fox & $29 / 223$ (13.0) & $\mathrm{Em}$ \\
\hline Sudan & Khartoum $^{48}$ & Stray dog & $40 / 84(47.6)$ & Ec (39); Eg (1) \\
\hline \multirow[t]{3}{*}{ Uzbekistan } & \multirow[t]{3}{*}{ Samarkand $^{49}$} & Wild fox & $0 / 5(0)$ & - \\
\hline & & Domestic dog & $24 / 1,749(1.4)$ & $\mathrm{Eg}$ \\
\hline & & Stray dog & $4 / 6(66.7)$ & $\mathrm{Eg}$ \\
\hline
\end{tabular}

$\mathrm{Em}=$ Echinococcus multiloculosus; Ec = Echinococcu canadensis; Eg = Echinococcu granulosus; Es = Echinococcu shiquicus; Eo = Echinococcus ortleppi.

*This study. - indicates no Echinococcus species test. 
on predatory wild rodents rather than on the abandoned organs of slaughtered cattle or sheep from human settlements. Thus we presume that compared with wild foxes, stray dogs may pose a greater risk to humans with regard to $\mathrm{AE}$ and should thus be the focus of future transmission prevention and control within wildlife cycles. Meanwhile, we should not neglect the role of wild foxes in Em transmission. In Haixi, animal husbandry is a relatively small contributor to the economy, and fewer stray dogs are present. Previous studies identified Haixi as a region of low CE prevalence, with a complete absence of $A E{ }^{17}$ Thus, no fecal samples were collected from stray dogs in this area, which may be one reason for the low prevalence of echinococcosis. Further, we did not observe Em in wild foxes from Haixi.

Several Es-positive samples from wild foxes were identified in all three regions. It is worth noting that Es was also detected in two stray dogs from Dari county in Golog, consistent with a previous study conducted in Sichuan. ${ }^{33}$ Interestingly, this pre-dates the detection of $\mathrm{Em}$ in plateau pika (Ochotona curzoniae) in Golog. ${ }^{11,12,34}$ To our knowledge, the current work represents the first report of Es in dogs from Qinghai province. Es is generally considered nonpathogenic to humans, and the epidemiological significance of dogs as definitive hosts remains to be determined. ${ }^{26} \mathrm{Eg}$ was not observed in wild fox fecal samples, which is consistent with a study conducted in Shiqu county, Sichuan province. ${ }^{23}$ In Australia, Eg has been reported to infect red foxes. ${ }^{35}$ However, foxes are not considered definitive hosts of $\mathrm{Eg}$ in China. ${ }^{5}$ In fact, the infection rates of parasites based on fecal samples are influenced by various factors, including the sampling locus, sample status, and detection methods. Nonetheless, positivity rates can still reflect the prevalence and distribution characteristics of parasitic diseases.

We reviewed literature to understand the prevalence of Echinococcus spp. worldwide and have summarized the findings in Table 3. The overall prevalence of Echinococcus spp. in definitive hosts varies globally. In China, the prevalence of Echinococcus spp. in domestic dogs has received considerable attention, but there have only been a few recent studies on Echinococcus spp. infection in wild foxes and stray dogs, which is of equal importance for assessing the risk of transmission to humans. Furthermore, previous studies largely focused on comparisons between detection methods, or are limited by small sample sizes for species identification. ${ }^{23-25,33}$ Therefore, systematic epidemiological studies for the prevalence of Echinococcus spp. in wild canines are of great importance. In the current study, the prevalence of Echinococcus species in wild foxes and stray dogs was determined, providing an overview of prevalence rates in wild canines across various endemic regions throughout Qinghai province. Consequently, our results provide valuable insight into Echinococcus infection in wild foxes and stray dogs in highly endemic area of China as well as a basis for risk assessment and the development of control and prevention methods.

\section{CONCLUSION}

Our results demonstrate that Qinghai province has a high prevalence of Echinococcus spp. (specifically, Em, Es, and $\mathrm{Eg}$ ) in wild foxes and stray dogs. These findings are crucial for the control and prevention of echinococcosis in the region. Further molecular characterization is essential to better understand the distribution and diversity of Echinococcus spp.

Received June 3, 2021. Accepted for publication September 29, 2021.

Published online November 15, 2021.

Acknowledgments: We thank the staff of the Center for Disease Control in Maqin, Dari, Banma, and Jiuzhi counties of Golog Prefecture; Yushu, Chenduo, and Zhiduo counties of Yushu Prefecture; and Delinha, Wulan, Dulan, and Tianjun counties of Haixi Prefecture for assistance in specimen collection.

Financial support: This work was supported by the health commission of Qinghai Province (Grant no. 2020-wjzd-15 to H. C.); the National Natural Science Foundation of China (Grant nos. 81772224 and 82072307 to YS); and NHC Key Laboratory of Parasite and Vector Biology China (Grant no. WSBKTKT201404 to HC).

Authors' addresses: Huixia Cai, Xuefei Zhang, Xiao Ma, Junying Ma, $\mathrm{Na}$ Liu, Yufang Liu, Jia Liu, Wei Wang, Wen Lei, Kemei Shi, Qing Zhang, Xiongying Zhang, and Peizhen Zhan, Department of Parasite Control, Qinghai Province Institute for Endemic Diseases Prevention and Control, Xining, China, E-mails: huixia_1107@163.com, 1730326847@qq.com, maxiao0971@163.com, mjy70315@163.com, 1060188129@qq.com, 289192520@qq.com, 457831207@qq.com, wwqhxn@126.com, 475986917@qq.com, 534663774@qq.com, 562709807@qq.com, 1510582718@qq.com, and 38069136@qq. com. Jing Zhang, Yayi Guan, Jianping Cao, and Yujuan Shen, National Institute of Parasitic Diseases, Chinese Center for Disease Control and Prevention, NHC Key Laboratory of Parasite and Vector Biology, WHO Collaborating Center for Tropical Diseases, National Center for International Research on Tropical Diseases, Shanghai, China, E-mails: silkfan@126.com, guan_ml@126.com, caojpcdc@ 163.com, and shenyj@nipd.chinacdc.cn. Hao Wu, Department of Medical Record Information, Qinghai Provincial Traffic Hospital, Xining, China, E-mail: 38992612@qq.com.

This is an open-access article distributed under the terms of the Creative Commons Attribution (CC-BY) License, which permits unrestricted use, distribution, and reproduction in any medium, provided the original author and source are credited.

\section{REFERENCES}

1. Mora P, Schantz PM, 2009. Echinococcosis: a review. Int $J$ Infect Dis 13: 125-133.

2. Nakao M, Lavikainen A, Yanagida T, Ito A, 2013. Phylogenetic systematics of the genus Echinococcus (Cestoda: Taeniidae). Int J Parasitol 43: 1017-1029.

3. Zhang T et al., 2014. Genetic characterization of human-derived hydatid cysts of Echinococcus granulosus sensu lato in Heilongjiang Province and the first report of G7 genotype of $E$. canadensis in humans in China. PLOS ONE 9: e109059.

4. Yang D, Zhang T, Zeng Z, Zhao W, Zhang W, Liu A, 2015. The first report of human-derived G10 genotype of Echinococcus canadensis in China and possible sources and routes of transmission. Parasitol Int 64: 330-333.

5. Wang ZH, Wang XM, Liu XQ, 2008. Echinococcosis in China, a review of the epidemiology of Echinococcus spp. EcoHealth 5: 115-126.

6. Qiu JM, Chen XW, Ren M, Luo CX, Liu DL, Liu XT, He DL, 1995. Epidemiological study on alveolar hydatid disease in Qinghai-Tibetan Plateau. J Pract Parasit Dis 3: 106-109 [in Chinese].

7. Ministry of Health, China, 2007. Report on the National Survey of Current Status of Major Human Parasitic Diseases in China. Beijing, China: People's Health Publishing House.

8. Wang GQ, Yu JJ, Wang Y, 2016. Epidemiological Survey on Echinococcosis in China. Shanghai, China: Shanghai Scientific \& Technical Publishers.

9. $\mathrm{Ma} X$ et al., 2017. Epidemiological investigation on hydatid disease/echinococcosis in Guoluo Tibetan autonomous prefecture in Qinghai Province. Chin J Parasitol Parasit Dis 35: 366-370 [in Chinese]. 
10. Chen SL et al., 2016. An epidemiological survey on echinococcosis in Yushu prefecture of Qinghai province. Chin J Parasitol Parasit Dis 34: 1-5 [in Chinese].

11. Han XM, Wang H, Cai HX, Ma X, Liu YF, Wei BH, Ito A, Craig PS, 2009. Epidemiological survey on echinococcosis in Darlag County of Qinghai Province. Zhongguo Ji Sheng Chong Xue Yu Ji Sheng Chong Bing Za Zhi 27: 22-26 [in Chinese].

12. Han XM, Wang H, Qiu JM, Ma X, Cai HX, Liu PY, Ding QJ, Dai $\mathrm{N}$, Ito A, Craig PS, 2006. An epidemiological survey on echinococcosis in Banma County of Qinghai Province. Chin $J$ Zoonoses 22: 189-190 [in Chinese].

13. Wu XH et al., 2007. Epidemiologic survey and studies on echinococcosis in humans in Jiuzhi county of Qinghai province. Chin J Zoonoses 23: 813-815 [in Chinese].

14. Ma X et al., 2015. Survey on Echinococcosis in Maqing County of Qinghai Province. Chin J Parasitol Parasit Dis 33: 269-272 [in Chinese].

15. Wu XH et al., 2007. An epidemiological survey on Echinococcosis in Zhiduo County of Qinghai province. Chin J Parasitol Parasit Dis 25: 229-231 [in Chinese].

16. Ma JY et al., 2005. Epidemiologic survey and studies on echinococcosis in humans in Yushu county of Qinghai province. Chin J Parasitol Parasit Dis 23: 452 [in Chinese].

17. Department of Disease Control, Ministry of Health, Chinese Center for Disease Control and Prevention and National Institute of Parasitic Diseases, C.D.C. China, 2008. A National Survey on Current Status of the Important Parasitic Diseases in Human Population. Beijing, China: People's Medical Publishing House.

18. Wang $Q$ et al., 2014. Review of risk factors for human echinococcosis prevalence on the Qinghai-Tibet Plateau, China: a prospective for control options. Infect Dis Poverty 3: 3.

19. Wang $\mathrm{H}, 2004$. Analysis on risk factors of human hydatidosis in Qinghai province. Chin J Parasitic Dis Contr 17: 214-216 [in Chinese].

20. Cui $X Y$ et al., 2020. The state of and risk factors for an Echinococcus infection in domestic dogs in Maqin County, Qinghai province. J Pathogen Biol 15: 692-697 [in Chinese].

21. Deplazes P, Eckert J, 1996. Diagnosis of the Echinococcus multilocularis infection in final hosts. Appl Parasitol 37: 245-252.

22. Wayne RK, Geffen E, Girman DJ, Koepfli KP, Lau LM, Marshall CR, 1997. Molecular systematics of the Canidae. Syst Biol 46: 622-653.

23. Jiang W, Liu N, Zhang G, Renqing P, Xie F, Li T, Wang Z, Wang $X, 2012$. Specific detection of Echinococcus spp. from the Tibetan fox (Vulpes ferrilata) and the red fox (V. vulpes) using copro-DNA PCR analysis. Parasitol Res 111: 1531-1539.

24. Liu CN et al., 2015. Discrimination between $E$. granulosus sensu stricto, E. multilocularis and E. shiquicus using a multiplex PCR assay. PLoS Negl Trop Dis 9: 1-14.

25. Nakao M, Sako Y, Yokoyama N, Fukunaga M, Ito A, 2000. Mitochondrial genetic code in cestodes. Mol Biochem Parasitol 111: 415-424.

26. Nakao M et al., 2010. Genetic polymorphisms of Echinococcus tapeworms in China as determined by mitochondrial and nuclear DNA sequences. Int $J$ Parasitol 40: 379-385.

27. Nakao M, McManus DP, Schantz PM, Craig PS, Ito A, 2007. A molecular phylogeny of the genus Echinococcus inferred from complete mitochondrial genomes. Parasitology 134: 713-722.

28. Torgerson PR, Robertson LJ, Enemarkx HL, Foehr J, van der Giessen JW, Kapel CM, Klun I, Trevisan C, 2020. Source attribution of human echinococcosis: a systematic review and meta-analysis. PLoS Negl Trop Dis 14: e0008382.

29. He DL, 2000. Epidemiology of echinococcosis, Qinghai, China. Chin J Zoonoses 16: 101-103 [in Chinese].

30. The Central People's Government of the People's Republic of China, 2010. Action Plan for Echinococcosis (2010-2015). Available at: http://www.gov.cn/zwgk/2010-12/14/content_ 1765485.htm.

31. Cai HX, Wang H, Han XM, Ma X, 2012. Correlation between definitive hosts of Echinococcus and echinococcosis in children in Qinghai Plateau, China, 1990-2010. Chin J Zoonoses 28: 500-502 [in Chinese].

32. Wei SH, Wu WP, Han S, Xue CZ, Liu BX, Wang X, Gong WC, Cui XY, Fu MH, 2020. Echinococcus eggs in canine feces in wild areas of Maqin County, Qinghai province. $J$ Pathogen Biol 15: 568-575 [in Chinese].

33. Boufana B, Qu JM, Chen XW, Budke CM, Campos-Ponce M, Craig PS, 2013. First report of Echinococcus shiquicus in dogs from eastern Qinghai-Tibet plateau region, China. Acta Trop 127: 21-24.

34. Xiao N, Qiu J, Nakao M, Li T, Yang W, Chen X, Schantz PM, Craig PS, Ito A, 2006. Echinococcus shiquicus, a new species from the Qinghai-Tibet plateau region of China: discovery and epidemiological implications. Parasitol Int 55: S233-S236.

35. Jenkins DJ, 2006. Echinococcus granulosus in Australia, widespread and doing well! Parasitol Int 55: S203-S206.

36. Liu CN et al., 2018. Estimating the prevalence of Echinococcus in domestic dogs in highly endemic for echinococcosis. Infect Dis Poverty 7: 77.

37. Hao LL, Yang AG, Yuan DB, Guo L, Hou W, Mo Q, Lu ZP, Nie CY, 2018. Detection of Echinococcus multilocularis in domestic dogs of Shiqu County in the summer herding. Parasitol Res 117: 1965-1968.

38. Li B et al., 2019. Epidemiological survey of echinococcosis in Tibet Autonomous Region of China. Infect Dis Poverty 8: 29.

39. Chen XY, Setiwaldi Y, Shi AH, Zhao L, Yisilayin O, 2016. Epidemiological investigation on Echinococcus granulosus infections in dogs and livestock in Xinjiang Kizilsu Kirgiz Autonomous Prefecture. J Trop Dis Parasitol 14: 195-197.

40. Thapa NK, Armua-Fernandez MT, Kinzang D, Gurung RB, Wangdi P, Deplazes P, 2017. Detection of Echinococcus granulosus and Echinococcus ortleppi in Bhutan. Parasitol Int 2: $139-141$.

41. Kotwa JD, Isaksson $M$, Jardine $C M$, Campbell GD, Berke $O$, Pearl DL, Mercer NJ, Osterman-Lind E, Peregrine AS, 2019. Echinococcus multilocularis infection, southern Ontario, Canada. Emerg Infect Dis 25: 265-272.

42. Grech-Angelini S, Richomme C, Peytavin de Garam C, Boucher $J M$, Maestrini $O$, Grenouillet F, Casabianca F, Boué $F$, Umhang G, 2019. Identification and molecular characterization of Echinococcus canadensis G6/7 in dogs from Corsica, France. Parasitol Res 118: 1313-1319.

43. Ingole RS, Khakse HD, Jadhao MG, Ingole SR, 2018. Prevalence of Echinococcus infection in dogs in Akola district of Maharashtra (India) by Copro-PCR. Vet Parasitol Reg Stud Rep 13: 60-63.

44. Mirbadie SR, Kamyabi H, Mohammadi MA, Shamsaddini S, Harandi MF, 2018. Copro-PCR prevalence of Echinococcus granulosus infection in dogs in Kerman, south-eastern Iran. J Helminthol 92: 17-21.

45. Mulinge E et al., 2018. Molecular characterization of Echinococcus species in dogs from four regions of Kenya. Vet Parasitol 255: 49-57.

46. Karamon J, Sroka J, Dąbrowska J, Bilska-Zając E, Zdybel J, Kochanowski M, Różycki M, Cencek T, 2019. First report of Echinococcus multilocularis in cats in Poland: a monitoring study in cats and dogs from a rural area and animal shelter in a highly endemic region. Parasit Vectors 12: 313.

47. Miljević M, Bjelić Čabrilo O, Simin V, Čabrilo B, Miljević JB, Lalošević D, 2019. Significance of the red fox as a natural reservoir of intestinal zoonoses in Vojvodina, Serbia. Acta Vet Hung 67: 561-571.

48. Omer RA, Daugschies A, Gawlowska S, Elnahas A, Kern P, Bashir S, Ali MSA, Osman A, Romig T, 2018. First detection of Echinococcus granulosus sensu stricto (G1) in dogs in central Sudan. Parasitol Res 117: 1657-1661.

49. Yong TS, Lee KJ, Shin MH, Yu HS, Suvonkulov U, Sergeevich TB, Shamsiev A, Park GM, 2019. Prevalence of intestinal helminth infections in dogs and two species of wild animals from Samarkand region of Uzbekistan. Korean J Parasitol 57: 549-552. 